\title{
Advanced Hardware Protection of Metallic Loops
}

\author{
Václav $\mathrm{MACH}^{1, *}$ and Milan ADÁMEK ${ }^{1}$ \\ ${ }^{1}$ Tomas Bata University in Zlín, Faculty of Applied Informatics, Nad Stráněmi 4511, 76005 Zlín, Czech Republic \\ *v2mach@fai.utb.cz
}

\begin{abstract}
This article deals with the advanced protection of metallic loops using addition electronic components. The main goal of this article is to improve especially Advanced Technology Zone (ATZ), which is the most applied method. The improvement consists of protection against overvoltage which can destroy the Control and Indicating Equipment (CIE) and it consist of adding more states which have the impact of numbers of connected detectors. All ideas are introduced with technical details, schematic and every idea also comes with an explanation.
\end{abstract}

\section{Introduction}

The Intruder Alarm System (IAS) is a part of technical security which can indicate the intrusion to the protected building or area. The concept is driven by the standard CSN CLC/TS 50131. The standard is divided into several parts that describe single areas. The most important standard for this article is the CSN CLC/TS 50131-1 Control and indicating equipment (CIE), which consist of information and parameters which every product must accomplish.

The CIE is the main component of the IAS. It consists of several components which are responsible for each function. These functions are:

- It receives and evaluates the signals from the detectors.

- It provides the power to the connected detectors and other equipment

- It allows system diagnostics

- It controls signalling, transmission, recording, and other functions that indicate the current conditions.

According to the evaluation of incoming signal, the commercial CIE can be divided into two groups. First group using digital interface and second using analog interface. Digital CIE using only one bus which typically consists of four cables. This manner allows to connecting several detectors to one bus. Detector evaluates actual state which is then sent to the CIE in binary form. [1]

CIE using analog loops evaluates actual state of each zone. Each loop has the maximum amount of connected devices according to used mode. These modes are Normal Closed (NC), End of Line (EOL) and ATZ. The most used mode is ATZ because it provides the biggest amount of possible connected detectors from all mentioned modes. Even nowadays ATZ mode is still commonly used in many applications.
ATZ mode still has many disadvantages and possible threats. One of the biggest disadvantages is the number of connected devices which is limited usually up to four devices. [2] This is given by a huge tolerance of terminating resistor which is usually $30 \%$. It means that states between each other must have a gap. This situation helps with the selection of terminating resistor but it inefficiently uses the working range.

The biggest threat is that every commercial CIE has only basic protection against overvoltage which can destroy CIE by the potential intruder. This protection is done using one-use fuse which is installed in each loop. [3] When higher voltage passes the fuse it becomes interrupted. The interrupted loop is not working anymore. In this case destroyed fuse must be replaced.

This article deals with mentioned disadvantages and it tries to bring a new solution. For purposes of this research, a prototype of new CIE was created. Created design is listed in following chapters.

\section{Advanced technology Zone}

ATZ mode uses different values of resistors. Different values can very precisely distinguish between individual detectors. Each detector has a resistor with a unique value. In real operation, multiple detectors are connected into several groups. Each group uses the same value of resistors.

In general, the ATZ variant has four states, but each individual group can be distinguishable. It can be done by using different values of resistors. This variant is limited by the final numbers of used groups. Usual ranges of values are R1 $=10 \mathrm{k} \Omega, \mathrm{R} 2=30 \mathrm{k} \Omega$ and $\mathrm{R} 3=$ $100 \mathrm{k} \Omega$. Adding more resistors can result in difficulties in distinguishing between groups. Each group can contain up to three detectors.

Every loop should be able to distinguish between basic states. These states for every variant are as follows: 
- Serenity

- Alarm

- Failure

- Sabotage

Serenity and Alarm state are very easy to identify. The tricky part comes with the detection and subsequent distinction between Failure and Sabotage. In the NC variant it is almost impossible to distinguish them. In such case, Failure must be detected as Alarm.

The connected detectors can have some additional outputs like tamper or anti-masking. These connections must be wired in the correct way according to the device manual and also an appropriate version must be used.

\section{Designed Hardware Protection}

Every analog CIE consists of Analog to Digital Converter (ADC) which converts the analog signal to the binary form. Usually, this converter is already embedded into the microcontroller. Every possible protection must be done outside of the microcontroller.

Designed protection consists of output part and input part. Input part should process not only the continuous signal but it also should process binary pattern. This pattern provides better protection against sabotage because is almost impossible to imitate random binary signal which is generated by the CIE. Due to this situation, every output of the CIE can be controlled. The control part is galvanically separated using an optocoupler to protect CIE from any other threats.

The diode in the forward direction is placed in the circuit to protect also the power supply against overvoltage. The diode is dimensioned up to $1 \mathrm{kV}$. Another active protection is the Transient Voltage Suppression (TVS) diode in combination with the resettable fuse. [4] TVS diode acts as a normal diode with the threshold voltage. In this case threshold voltage is set to $11 \mathrm{~V}$. When this voltage is exceeded diode becomes to the short circuit mode and voltage is passed to the Ground (GND). [5] In the same time resettable fuse disconnects the circuit due to the big current from the external power supply. TSV diode can turn back from the short circuit state after the voltage drops back under $11 \mathrm{~V}$. Also the resettable fuse can turn back to the connected state. It means that this protection is nondestructive. Schematic can be found in the following figure.

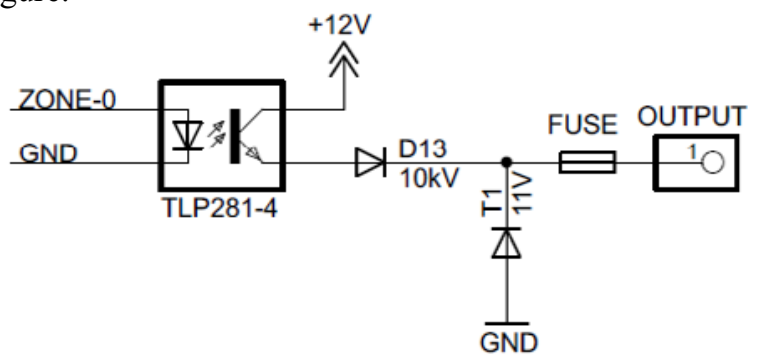

Fig. 1. Output part of created protection of analog loop.

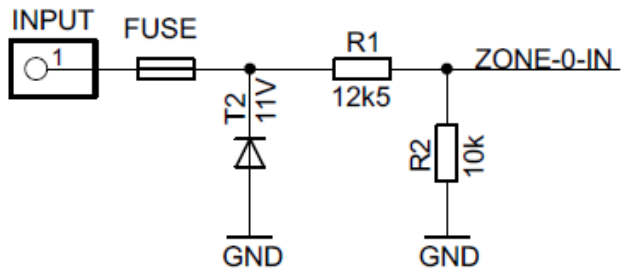

Fig. 2. Input part of created protection of analog loop.

The input part consists of a twin set of TVS diode and resettable fuse as well as the output part. Before voltage gets to the ADC it passes through the voltage divider. Operating voltage of the circuit is $10,9 \mathrm{~V}$. But the maximal voltage level which can be applied to the $\mathrm{ADC}$ is only $5 \mathrm{~V}$. The entering voltage must be lowered using the voltage divider.

Voltage divider uses resistors with values $10 \mathrm{k} \Omega$ and $12,5 \mathrm{k} \Omega$. It means that output voltage of the voltage divider is 4,84 which is safe for the ADC built in the microcontroller. This precise value is used to evaluate short circuit state of the loop. All possible states are listed in Table 2.

All modes use NC contact and resistor in the loop. When the detector is activated, it opens the NC contact. It causes that connected resistor is no long bypassed and the resistor is connected to the loop. ATZ mode uses several resistors with different values to identify, which of these resistors were connected to the loop. The total value of the resistor in the loop is changing by adding more resistor to it. This change is detected and evaluated in the CIE.

Own values of resistors were uses to achieve the satisfying number of the possible detectors connected to the loop. Used values are listed in Table 1. These values are not very common but all these values can be found in the market. As mentioned before, commercial tolerance for the resistor value is about $30 \%$. [6] Listed values of the resistor must have only $5 \%$ tolerance to achieve maximal states without interference or overlapping. Created schematic with detectors and resistor can be found in the following figure.

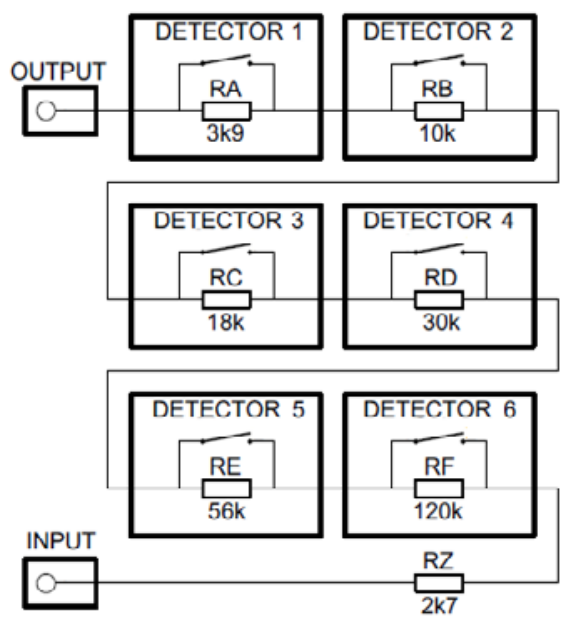

Fig. 3. Resistor values using ATZ mode. 
Before final evaluation, entering voltage must be converted using ADC. Used ATmega2560 the microcontroller has a built-in 1024-bit converter. It means that range of entering voltage is converted into numbers from 0 to 1023. Conversion values are shown in the following table.

Table 1. Calculated values of resistors.

\begin{tabular}{|c|c|c|c|}
\hline $\begin{array}{c}\text { Current } \\
\text { State }\end{array}$ & $\begin{array}{c}\text { Value } \\
\text { of ADC }\end{array}$ & $\begin{array}{c}\text { Input } \\
\text { voltage }\end{array}$ & $\begin{array}{c}\text { Active } \\
\text { resistor }\end{array}$ \\
\hline- & {$[-]$} & {$[\mathrm{V}]$} & {$[\mathrm{k} \Omega]$} \\
\hline Short-circuit & 1023 & 4,84 & 0 \\
\hline Serenity & 915 & 4,33 & $2 \mathrm{k} 7$ \\
\hline Active - RA & 792 & 3,75 & $3 \mathrm{k} 9+\mathrm{RZ}$ \\
\hline Active - RB & 655 & 3,10 & $10 \mathrm{k}+\mathrm{RZ}$ \\
\hline Active - RC & 534 & 2,52 & $18 \mathrm{k}+\mathrm{RZ}$ \\
\hline Active - RD & 418 & 1,97 & $30 \mathrm{k}+\mathrm{RZ}$ \\
\hline Active - RE & 284 & 1,34 & $56 \mathrm{k}+\mathrm{RZ}$ \\
\hline Active - RF & 159 & 0,75 & $120 \mathrm{k}+\mathrm{RZ}$ \\
\hline Sabotage & 0 & 0 & Infinity \\
\hline
\end{tabular}

Selected values of used resistors have an exponential rising tendency. This tendency helps to achieve a linear order of resulting voltage. Created voltage levels are shifted from each other by $0,5 \mathrm{~V}$. Created gap and 5\% tolerance helps against any overlapping or distortion.

Created design needs also software extension. Used ATmega2560 the microcontroller allows using several programming platforms such as Atmel Studio or Arduino. Values of the ADC from Table 1 can be used as threshold values for the software extension. The program should consist of all possible states which are mentioned in Table 1.

\section{Measurement}

The experiment was done in the laboratory of Tomas Bata University in Zlín at Faculty of Applied Informatics. Used components are listed below.

- ATmega2560 the microcontrollers

- Digital multi-meter M-3900

- Power supply LBN-303 0-30V/0-3A

The Printed Circuit Board (PCB) was created, to confirm that designed system is working. Tested PCB was connected to the power supply. The pin named ZONE-0 was set to logic 1 using the test program. This change causes that optocoupler activated the loop. Each resistor was added to the loop and by the ADC in the microcontroller entering voltage was measured and displayed.

The measurement was primarily focused on the independent states, which can occur. Measured values are presented in the following Figure 4. The measurement shows designed levels of each state. Presented states are given by the resistor values in Table 1.

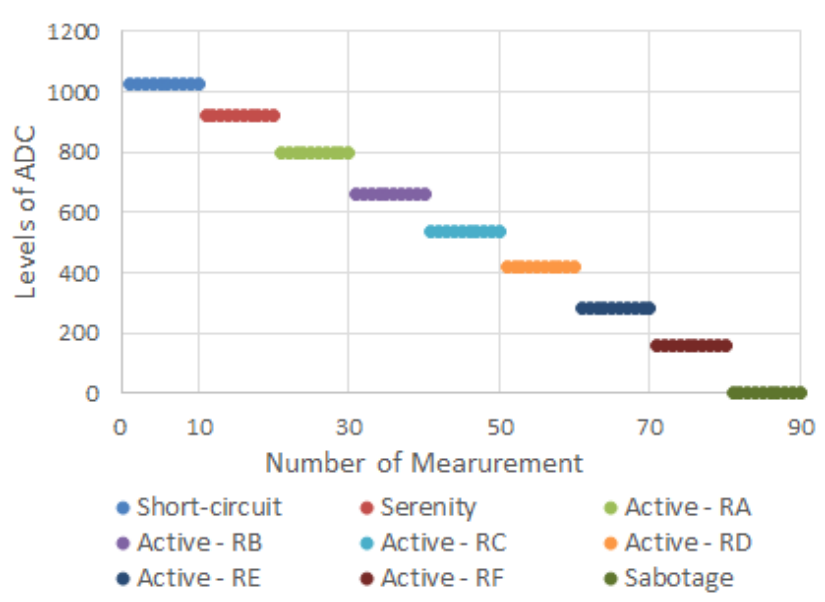

Fig. 4. Measurement results of each state.

Ten measurements of each state were taken to obtain a satisfactory amount of data. Even with this amount of values, states are very stabilized according to calculated values. From Figure 4 can be seen the gap between each state to ensure minimal possible distortion or overlapping. Another measurement was done according to the protection against overvoltage using TVS diode and resettable fuse.

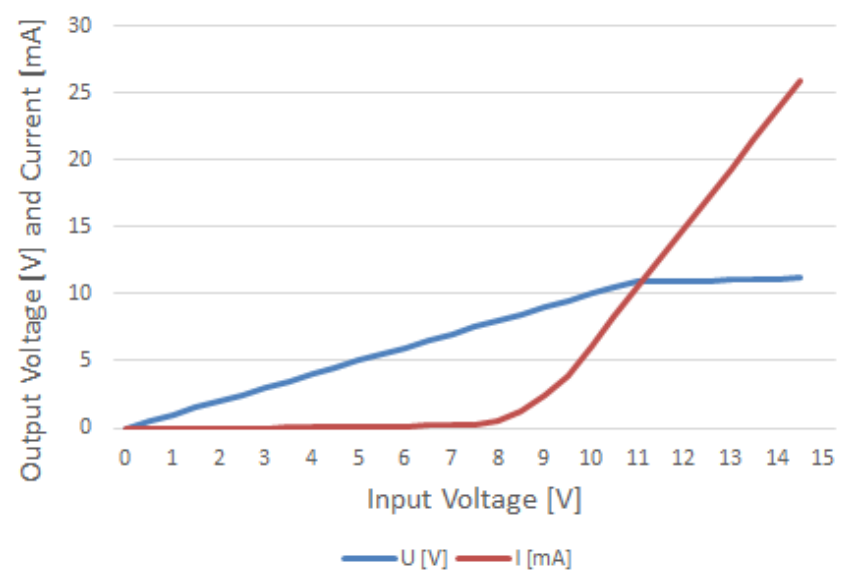

Fig. 5. Measurement results of TVS diode.

Previous Figure 5 shows the situation when the overvoltage occurs. In this case, the input voltage is limited by the TVS diode. This diode is dimensioned to the $11 \mathrm{~V}$. When this voltage is exceeded TVS diode slowly becomes to the short circuit state. It means that voltage does not rise, but the current does. When current reach value approximately to $20 \mathrm{~mA}$ resettable fuse opens the circuit. Resettable fuse activates approximately at $13.5 \mathrm{~V}$. This level protects CIE against overvoltage very well.

\section{Conclusion}

Created design adds to the CIE more electrical protection against sabotage by the overvoltage and it also allows to use more possible levels. All presented schematics were assembled and then tested. These measurements are 
listed and commented in several figures. To personal safety, the sabotage by overvoltage was tested only to 15 $\mathrm{V}$. But even $15 \mathrm{~V}$ proved that designed schematic works. All measurements were done using the 8-bit ADC embedded in ATmega2560 the microcontroller. The TVS diode was tested using Mastech-M3900 digital multi-meter.

This work was supported by the Ministry of Education, Youth and Sports of the Czech Republic within the National Sustainability Programme Project No. LO1303 (MSMT7778/2014) and also by the European Regional Development Fund under the project CEBIA-Tech No. CZ.1.05/2.1.00/03.0089 and by the Internal Grant Agency of Tomas Bata University under the project No. IGA/CebiaTech/2017/006.

\section{References}

1 D. Brooks. S.J., 24 (2), 101-117, (2011)

2 A. Hanáček, M. Sysel. S.V., 119-128, (2015)

3 I. Strašil. B.U.T. (2008)

4 D. Bouangeune, D. Cho, H. Yun, K. Shim, CH.O.I.

11, 88-92, (2015)

5 V. Mach. T. 5 (2), (2016) 